\title{
Antioxidant effect of flavonoids in neurodegenerative diseases
}

\author{
Adriana E. Bulboaca', Sorana S. Bolboaca², Ioana C. Stanescu³, \\ Carmen A. Sfrangeu ${ }^{1}$, Angelo C. Bulboaca ${ }^{3}$ \\ ${ }^{1}$ Department of Pathophysiology, "Iuliu Hatieganu" University of Medicine and Pharmacy, \\ Cluj-Napoca, Romania \\ ${ }^{2}$ Department of Medical Informatics and Biostatistics, \\ "Iuliu Hatieganu" University of Medicine and Pharmacy, Cluj-Napoca, Romania \\ ${ }^{3}$ Department of Neurology and Pediatric Neurology, \\ "Iuliu Hatieganu" University of Medicine and Pharmacy, Cluj-Napoca, Romania
}

\begin{abstract}
The investigation of the mechanisms that leads to neuronal apoptosis is under investigation all over the world. The enhancement of oxidative stress in brain neurons is one of the most important pathophysiological mechanism asso-ciated with neurodegeneration. The antioxidant effect of flavonoids can be one important mechanism for modulation of neuronal apoptosis in neurodegenerative diseases such as Alzheimer's and Parkinson's disease. In addition to their influence on the cerebral blood flow, flavonoids interact with signalization cascades that lead to the inhibition of neuronal death by oxidative stress apoptosis and thus promote neuronal survival and synaptic plasticity. Acting on the intracellular antioxidant mechanisms the flavonoids can become a balance regulator for production of reactive oxygen species (ROS) and reactive nitrogen species (RNS). Addition of antioxidant effect of flavonoids to dietary components can constitute an adjuvant therapy for delaying the neurodegenerative processes. The aim of this paper was to review the antioxidant effects of flavonoids in Alzheimer's and Parkinson's diseases.
\end{abstract}

Keywords: neurodegenerative disease, Alzheimer's disease, Parkinson's disease, flavonoids, Reactive Oxygen Species (ROS), antioxidant

\section{INTRODUCTION}

Flavonoids constitute a major group of polyphenolic compounds which are directly associated with the health-promoting properties (1). The group of flavonoids includes over 6,000 already-identified family members classified in several subgroups (such as anthocyanidins, flavans, flavanonols, flavanones, and anthoxanthins) (flavone and flavonol) (2). Flavonoids are found in fruits (apples, blueberries, cranberries) (3), vegetables (4), grains (5), herbs (6), as well as on chocolate (milk and dark) (7) and are known to have antioxidant and anti-inflammatory proprieties $(8,9)$. Furthermore, the effect of flavonoids as modulators of cardiovascular $\mathrm{Ca}_{\mathrm{v}}$ channels are investigated as poten- tial of development of new drugs (10). In vitro and in vivo experiments support the potential antidiabetic effect of some flavonoids but the efficacy on humans with diabetes have not been yet sufficiently investigated (11). George et al. showed the protective effect of flavonoids against DNA damage produce by various carcinogenic factors (12). The benefic effect of flavonoids on different types of malign tumors have also been investigated (13-16).

According with the epidemiological reports published by the World Health Organization, the proportion of elderly people (over 60 years) will increase from $11 \%$ to $22 \%$ by 2050 worldwide (17). Neurodegenerative diseases are important health problems in elderly people and they are pro- 
portionally related with the increasing of life expectancy. One of the most neuroprotective agents that have low adverse effects can be flavonoids and recent studies has been focused on finding effective neuroprotective agents based on their chemical compounds $(18,19)$. Polyphenols permeation through the blood brain barrier (BBB) as it was demonstrated using in vitro and this phenomenon is dependents on the degree of lipophilicity of each compound. Thus, less polar polyphenols or metabolites (i.e., $O$-methylated derivatives) are capable of greater brain uptake than the more polar ones (i.e., sulfated and glucuronidated derivatives) (20).

Accumulating evidence has demonstrated that flavonoids exert many protective properties, including those for neurodegenerative diseases due to their properties to cross the blood-brain barrier. Several antioxidant mechanisms have been proposed as being involved in antioxidant activity of flavonoids and they are related to activation of nuclear factor erythroid 2-related factor 2 (Nrf2) pathway, up regulation of antioxidant enzymes, induction of hypoxia signal transduction (HIF- 1 alpha pathway) and interaction with metal ions as sources of reactive oxygen species (ROS) (21). Thus, the identification of flavonoids targeting neuroprotective effect in neurodegenerative processes may represent an attractive approach to the more effective therapeutic strategies for diseases such as Alzheimer's (AD) or Parkinson's disease (PD) that have only few effective disease-modifying therapies. The objective of this mini-review was to analyze the results of studies concerning the flavonoids antioxidant effects in neurodegenerative diseases published in the last 10 years, comparing the results and emphasizing the actual stage of the knowledge.

\section{THE ROLE OF OXIDATIVE/NITROSATIVE STRESS IN NEURODEGENERATION}

Oxidative/nitrosative stress is a normal process of aerobic organisms that are maintained at low levels by several enzyme systems participating in the in vivo redox homeostasis. This process consists in the production of reactive oxygen species (ROS) and reactive nitrogen species (RNS). Production of low/moderate ROS or RNS can regulate the balance of body homeostasis (redox homeostasis) by exerting the effect of signal transduction role from membrane receptors in various physio- logical processes $(22,23)$. Both reactive species are produced by strictly regulated enzymes, such as nitric oxide synthase (NOS), and isoforms of NADPH oxidase, or as by-products from not so well regulated sources, such as the mitochondrial electron-transport chain (24). Excessive production of ROS and RNS have a negative effect contributing to the initiation and maintenance of oxidative/nitrosative stress that have a major contribution in neurodegenerative disease pathophysiology by damaging nucleic acids, lipids and proteins. These processes can severely compromise neurons health and viability, or can induce a variety of cellular responses through generation of secondary reactive species, finally causing to cell death by necrosis or apoptosis $(25,26)$. As sources of the majority of energy production and endogenous ROS production, mitochondria play a key role in the functioning and survival of neurons in the brain. Therefore, mitochondrial dysfunction can be a source of excessive production of ROS (27). Additionally, lipid peroxidation may contribute to and amplify cellular damage resulting from generation of oxidized products, some of which are chemically reactive and covalently modify critical macromolecules. Products of lipid peroxidation have therefore commonly been used as biomarkers of oxidative/nitrosative stress/ damage. Lipid peroxidation generates a variety of relatively stable decomposition end-products, mainly $\alpha, \beta$-unsaturated reactive aldehydes, such as malondialdehyde (MDA), 4-hydroxy-2-nonenal (HNE), 2-propenal (acrolein) and isoprostanes ( $\mathrm{F}_{2}-$ IsoPs) (1), which can then be measured in plasma and urine as an indirect index of oxidative/nitrosative stress. In AD brain, increased levels of amyloid beta $(A \beta)$ were found in the affected regions. Several lines of evidence indicate that $A \beta$ induces oxidative stress. The $A \beta_{1-42}$ (that is critical for $A D$ pathogenesis) inserts as oligomers into the bilayer and serves as a source of ROS, and initiates lipid peroxidation (28). A critical process that leads to the progressive neuropathology of neurodegeneration in Parkinson's disease is the prion-like trans-neuronal propagation of alpha-synuclein. The endocytosis of alpha-synuclein into neurons triggers abnormal protein aggregation leading to a cytotoxic cascade that culminates in mitochondrial dysfunction and cell death. The pathogenetic mechanisms where the biology of alpha-synuclein is altered 
by oxidative stress while oxidized or nitrated alpha-synuclein has greater neurotoxicity create a state of progressive neuronal death seen in PD $(29,30)$.

\section{FLAVONOIDS AND BLOOD BRAIN BARRIER PENETRATION}

Before oral absorption, flavonoids undergo deglycosylation either by lactase phloridzin hydrolase or cytosolic $\beta$-glucosidase. The absorbed aglycone is then conjugated by methylation, sulphatation or glucuronidation (31). Both the aglycones and the conjugates can pass the blood-brain barrier (20). Flavonoids of several classes are inhibitors of monoamine oxidase A or B, thereby working as anti-depressants or to improve the conditions of Parkinson's patients. Flavanols, flavanones and anthocyanidins have protective effects preventing inflammatory processes consequently acting as defense mechanisms against neuronal injury $(2,32)$. The blood-brain barrier (BBB) is mainly formed by brain capillary endothelial cells that have tight junctions, which prevent paracellular transport of small and large water-soluble compounds from blood to the brain. Other cell types such as pericytes, astrocytes and neuronal cells also play an important role. Transcellular transport is further limited due to low vesicular transport and high metabolic activity. The BBB play a role of a physical and metabolic barrier and aging can be associated with alteration of BBB structures and transport mechanisms. In vivo and in vitro studies demonstrated that the flavonoids have the ability to cross the BBB and the permeability for different structures can be different. One of these studies showed that the some molecules penetration of BBB by some studied molecules could be stratified as follow: naringenin $>$ hesperetin $>$ naringenin glucuronide $>$ hesperetin glucuronide $>$ cyaniding-3-rutinoside $>$ pelargonidin-3-glucoside (20). The in vivo studies show that flavonoids are able to be absorbed after oral administration, pass the blood-brain barrier and do have various effects on the CNS (33).

\section{ANTIOXIDATIVE MECHANISMS OF FLAVONOIDS IN NEURODEGENERATIVE DISORDERS}

The antioxidative pathophysiological mechanisms related to flavonoids has been studied. Sever- al evidences suggests that oxidative stress is related to the pathogenesis of Alzheimer's and Parkinson's neurodegenerative diseases. Because flavonoids may help to improve blood flow in the brain, there is also preliminary evidence to suggest the possibility of better brain functioning in some areas, including areas involving cognitive function (34). Literature evidences emphasized pathophysiological mechanisms involved and some of them are presented in this section.

\section{Flavonoids and Activation of Nuclear Related Factor 2 Pathway in the Brain}

Nuclear related factor $2(\mathrm{Nrf} 2)$ is a powerful protein that is latent within each cell in the body, that is released after activation by an Nrf2 activator. One of this activator can be represented by arachidonic acid production due to inflammation (35). Once released it migrates into the cell nucleus and bonds to the DNA at the location of the Antioxidant Response Element (ARE) (also called hARE $=\mathrm{Hu}$ man Antioxidant Response Element) which is the master regulator of the total antioxidant system that is available in all human cells. Nuclear factor -erythroid 2-related factor-2 (Nrf2) mediate the anti-inflammatory mechanism by regulation of cellular antioxidant production and play an important role in neuroprotection against neurodegenerative diseases.

Curcumin (derived from turmeric plant) demonstrated anti-oxidative properties (36), strongly induces heme-oxygenase-1 (HO-1) expression and activity in different brain cells via the activation of heterodimers of NF-E2-related factors 2 (Nrf2)/antioxidant responsive pathway. Activation of $\mathrm{Nrf} 2$ target genes, and particularly $\mathrm{HO}-1$, in astrocytes and neurons is strongly protective against inflammation, oxidative damage and cell death. Other phenolics, such as caffeic acid phenethyl ester and ethyl ferulate, are also able to protect neurons via HO-1 induction (37).

Genistein (an isoflavone found especially in soybeans) has antioxidative properties against neurodegeneration induced by beta-amyloid peptides, that has been proved to be associated with Alzheimer's disease. Some results showed that genistein induces the increase of reactive oxygen species (ROS) and nitrotyrosine production induced by $A \beta 25-35$, and maintained cell redox state by in- 
creasing GSH level and GSH/GSSG. Genistein could reverse the down-regulation of total protein and mRNA expression of NF-E2-related factor 2 (Nrf2), nuclear Nrf2, $\gamma$-glutamylcysteine synthetase $(\gamma$-GCS), phosphatidylinositol 3-kinase (PI3K) induced by A $\beta 25-35$. These results suggested that genistein could protect cerebrovascular endothelial cells from beta- amyloid induced oxidative damage by activation of Nrf2 signaling pathway (38).

Naringin (a citrus flavonone) induces NAD $(\mathrm{P})$ $\mathrm{H}$ : quinone oxido reductase-1, heme oxygenase-1, glutathione $\mathrm{S}$ - transferase $\mathrm{P} 1$ and gamma-glutamylcysteine ligase mRNA expressions through the activation of Nrf2 and decreased the expressions of pro-inflammatory mediators TNF-alpha and COX2 (inflammation mediators). It also decrease the activity of and iNOS (enzymes that contribute to nitro-oxidative stress by induction of nitric oxide formation). These results indicate that naringin might be protective against neurodegeneration through the enhancement of phase II and antioxidant gene expressions via Nrf2 activation; thereby modulating the oxidative stress and inflammatory responses (39). Lou at al. also found that naringenin treatment resulted in an increase in nuclear factor E2-related factor 2 (Nrf2) protein levels and subsequent activation of antioxidant response element (ARE) pathway genes in SH-SY5Y culture cells and and mice exposed to experimental neurotoxicity (40). Exposure of SH-SY5Y cells to naringenin provided protection against 6-OHDA-induced oxidative insults that was dependent on Nrf2. In mice, oral administration of naringenin resulted in significant protection against 6-OHDA-induced nigrostriatal dopaminergic neurodegeneration and oxidative damage (40).

Puerarin, one of several known isoflavones, is found in a number of plants and herbs, such as the root of Pueraria significantly ameliorates cognitive impairment in Alzheimer's disease (AD) induced in mice assessed by the Morris water maze (MWM) test. This was accompanied by a significant decrease in the levels of lipid peroxidation through, at least in part, induction of nuclear factor erythroid 2-related factor 2 (Nrf2) target gene heme oxygenase 1 (HO-1) in the hippocampus. Furthermore, puerarin significantly induced nuclear translocation of Nrf2 in the hippocampus. These findings suggest that puerarin can contribute to prevention and treatment of cognitive impairment and dementia (41).

There was been found that the sulfuretin, a flavonoid glycosides found in the stem bark of Albizzia julibrissin and heartwood of Rhus verniciflua, known having anti-oxidant that the neuroprotective properties protects neuronal cells from $A \beta 25-35$ induced neurotoxicity through activation of $\mathrm{Nrf} /$ HO-1 and PI3K/Akt signaling pathways. Sulfuretin-induced induction of Nrf2-dependent HO-1 expression via the PI3K/Akt signaling pathway has preventive and/or therapeutic potential for the management of Alzheimer's disease (42).

Gaballah et al. found that activation of Nrf2 signaling pathway by resveratrol reduce oxidative stress by preserving intracellular antioxidant mechanisms in rat model for Parkinson's disease (43). Several other mechanisms were demonstrated: ameliorating of endoplasmic reticulum stress by down regulating CHOP and GRP78 genes expression and hampered caspase- 3 activity in the brain of rotenone induced PD in rats. It also restored redox balance as evident by suppressing xanthine oxidase activity and protein carbonyls formation. In addition, resveratrol contributes to preservation of intracellular antioxidants status via activating glutathione peroxidase (43). The effects induced by flavonoids through activation of Nrf2 in brain cells is presented in Table 1.

TABLE 1. Types of effects induced by flavonoids through activation of Nrf2 in brain cells

\begin{tabular}{|l|l|l|}
\hline Flavonoid & Associated anti-inflammatory/anti-oxidant mechanisms & Main author, year (ref) \\
\hline Genistein & ac va on of NF-E2 - related factor, Gamma- GCS, and PI-3K & Xi, 2012 (38) \\
\hline Naringenin & $\begin{array}{l}\text { induc on of NAD(P)H -quinone oxidoreductase 1, and hem-oxygenase } \\
\text { decrease expression of TNF-alpha, COX2, and iNOS }\end{array}$ & $\begin{array}{l}\text { Gopinath, 2012 (39) } \\
\text { Shakeel, 2017 (44) }\end{array}$ \\
\hline Puerarin & $\begin{array}{l}\text { down regula ng A } \beta_{1-40} \text { and Bax expression in brain ssue } \\
\text { decreasing the lipid peroxidase levels and increasing superoxide } \\
\text { dismutase levels }\end{array}$ & $\begin{array}{l}\text { Gao, 2013 (45) } \\
\text { Zhou, 2014 (41) }\end{array}$ \\
\hline Sulfure $\mathrm{n}$ & ac va on of HO-1 signaling pathway & Kwon, 2015 (42) \\
\hline Resveratrol & ac va on of intracellular an oxidant mechanisms & Gaballah, 2016 (43) \\
\hline
\end{tabular}




\section{Flavonoids and up Regulation of Antioxidant Enzymes in Brain Cells}

The relationship between the cellular consequences of oxidative stress and the progression of neurodegenerative disorders has undergone continued researches. Mitochondria-derived radical oxygen species (ROS) and nitrosative stress are clinically relevant as an important contributing factor for neurodegeneration in brain injury and chronic neurodegenerative disorders. Recently, increased interest was observed in other sources of ROS and nitric oxide syntase (NO) such as NADPH oxidase, nitric oxide (NOS), cytochrome450 (cyp450), cyclooxygenase, lipooxygenase, and xanthine oxidase. NOS activation can be increased by a variety of inflammatory and neurodegenerative factors, such as amyloid precursor protein (APP), amyloid $\beta$, tumor necrosis factor-alpha (TNF- $\alpha$ ), matrix metalloproteinase (MMP), interleukins, and $\alpha$-synucleins, contributing to neuronal damage and cell death (46).

The role of flavonoids in central nervous system neurons, both brain and spinal cord, has been demonstrated by several studies. The evidences that age related dementia and Alzheimer's disease may be delayed by long term intake of flavonoids were emphasized by several studies. The amyloid potential for aggregation was demonstrated to be inhibited by ten antioxidant flavonoids isolated from the medicinal halophyte Tamarix gallica. Glucuronosylated flavonoids show relatively strong inhibitory activity of amyloid $\beta(\mathrm{A} \beta)$ and human islet amyloid polypeptide (hIAPP) aggregation compared to their aglycone analogs. There has been found a strong correlation between chemical structure and activity of flavonoids that suggests that the catechol moiety is important for amyloid aggregation inhibition, while the methylation of the carboxyl group in the glucuronide moiety and of the hydroxyl group in the aglycone flavonoids decreased it. This study demonstrated that flavonoidactivity can be useful not only for metabolic disturbances that can concurred for Alzheimer's disease pathogenesis but either for diabetes mellitus type 2 (47). However, $A \beta$ toxicity can also be reduced by the inhibition of $A \beta$ deposition via the antioxidative activity of the soy isoflavone glycitein $(100 \mu \mathrm{g} / \mathrm{mL})$ (48). Apigenin ameliorates $\mathrm{AD}$-associated learning and memory impairment via inhibiting $A \beta$ deposition and decreasing insoluble $A \beta$ concentrations, inhibiting oxidative stress, and improving the antioxidative enzyme activity of superoxide dismutase (SOD) and glutathione peroxidase (49).

Several polyphenols including flavonoids such as baicalein, daidzein, luteolin and nobiletin as well as nonflavonoid polyphenols such as auraptene, carnosic acid, curcuminoids and hydroxycinnamic acid derivatives including caffeic acid phentyl ester enhance neuronal survival and promote neurite outgrowth in vitro, a hallmark of neuronal differentiation. These effects were correlated with antioxidative activity of these compounds that is related to up regulation of Nrf 2 and the consequent up regulation of detoxification enzymes such as heme oxygenase-1. Furthermore, these effects are contributing to the neurotrophic activity reducing neurodegenerative process (21). Certain dietary flavonoids (as in cocoa and green tea) are related to reducing the NADPH oxidase activity in brain and consequently reducing the nitro-oxidative stress (50). Resveratrol was found strongly to inhibit NO generation in activated macrophages by reducing the iNOS (inducible nitric oxide synthase) expression (51).

Duarte et al. showed that flavonoids exert complex actions on the synthesis and bioavailability of NO and several mechanisms may decreased NO levels (52). In cell free systems, flavonoids may scavenge NO via its pro-oxidant properties by increasing superoxide. However, under conditions of oxidative stress, flavonoids may also protect NO from superoxide-driven inactivation. Under conditions of inflammation and oxidative stress, flavonoids may prevent the inflammatory signaling cascades via inhibition of $\mathrm{NF} \kappa \mathrm{B}$ and thereby down regulate iNOS. They also prevent the over expression of ROS generating enzymes, reducing superoxide and peroxynitrite levels, and hence preventing superoxide-induced NO inactivation and INOS uncoupling (52).

\section{CONCLUDING REMARKS}

The pathogenesis of Alzheimer's and Parkinson's diseases is linked to oxidative stress which leads to neuronal death and dysfunctions as major consequences. The central cause of imbalance for 
oxidative stress enhancement is attributed to intracellular processes dysfunctions that seem to have a critical importance. Flavonoids proved to induce several antioxidative intracellular mechanisms such as activation of nuclear related factor 2 (Nrf2), and up regulation of antioxidant enzymes in brain cells, that are important mechanisms for modulation of oxidative/nitrosative stress. The beneficial role of natural products from diet such as flavonoids becomes extensively studied because of their ability to interfere with oxidative stress and de- crease the excessive production of ROS and RNS, influencing the neuronal death and neuronal degenerative diseases. Increased numbers of such studies can be helpful for developing long term strategies in the prophylaxis of neurodegenerative process associated to Alzheimer's and Parkinson's diseases.

Supplementary Materials: Supplementary materials can be found at www.mdpi.com/link.

Conflicts of Interest: The authors declare no conflict of interest.

Financial support: none declared

\section{REFERENCES}

1. Fernández S.P., Wasowski C., Loscalzo L.M., Granger R.E., Johnston G.A.R., Paladini A.C., Marder M. Central nervous system depressant action of flavonoid glycosides. European Journal of Pharmacology 2006, 539, 168-176.

2. Jäger A.K., Saaby L. Flavonoids and the CNS. Molecules 2011, 16, 1471-1485. doi:10.3390/molecules16021471

3. Singh B., Singh J.P., Kaur A., Singh N. Bioactive compounds in banana and their associated health benefits - A review. Food Chemistry 2016, 206, 1-11. doi: 10.1016/j.foodchem.2016.03.033

4. Mitchell A.E., Hong Y.J., Koh E., Barrett D.M., Bryant D.E., Denison R.F., Kaffka S. Ten-year comparison of the influence of organic and conventional crop management practices on the content of flavonoids in tomatoes. Journal of Agricultural and Food Chemistry 2007, 55(15), 6154-6159. doi:10.1021/jf070344+.

5. Birla D.S., Malik K., Sainger M., Chaudhary D., Jaiwal R., Jaiwal P.K. Progress and challenges in improving the nutritional quality of rice (Oryza sativa L.). Critical Reviews in Food Science and Nutrition 2017, 57(11), 2455-2481. doi: 10.1080/10408398.2015.1084992.

6. Justesen U., Knuthsen P. Composition of flavonoids in fresh herbs and calculation of flavonoid intake by use of herbs in traditional Danish dishes. Food Chemistry 2001, 73(2), 245-450. doi:10.1016/ S0308-8146(01)00114-5.

7. Beecher G.R. Overview of dietary flavonoids: nomenclature, occurrence and intake. J Nutr. 2003, 133(10), 3248S-3254S.

8. Jurikova T., Mlcek J., Skrovankova S., Balla S., Sochor J., Baron M., Sumczynski D. Black crowberry (Empetrum nigrum L.) flavonoids and their health promoting activity. Molecules 2016; 21(12): Article number 1685. doi: 10.3390/molecules21121685

9. Zhao Y., Zhao K., Jiang K., Tao S., Li Y., Chen W., Kou S., Gu C., Li Z., Guo L., White W.L., Zhang K.X. A review of flavonoids from Cassia species and their biological activity. Current Pharmaceutical Biotechnology 2016, 17(13), 1134-1146. doi: 10.2174/138920101766 6160819151153

10. Fusi F., Spiga O., Trezza A., Sgaragli G., Saponara S. The surge of flavonoids as novel, fine regulators of cardiovascular Cav channels. European Journal of Pharmacology 2017, 796, 158-174. doi: 10.1016/j.ejphar.2016.12.033

11. Eid H.M., Haddad P.S. The antidiabetic potential of quercetin: Underlying mechanisms. Current Medicinal Chemistry 2017, 24(4), 355-364. doi: 10.2174/0929867323666160909153707

12. George V.C., Dellaire G., Rupasinghe H.P.V. Plant flavonoids in cancer chemoprevention: role in genome stability. Journal of Nutritional Biochemistry 2017, 45, 1-14. doi: 10.1016/j. jnutbio.2016.11.007.

13. George V.C., Vijesh V.V., Amararathna D.I.M., Lakshmi C.A., Anbarasu K., Kumar D.R.N., Ethiraj K.R., Kumar R.A. Vasantha Rupasinghe, H.P. Mechanism of action of flavonoids in prevention of

inflammation-associated skin cancer. Current Medicinal Chemistry 2016, 23(32), 3697-3716. doi: $10.2174 / 0929867323666160627110342$

14. Wang B., Wang J., Zhao X.-H. In vitro Activities of the Four Structurally Similar Flavonols Weakened by the Prior Thermal and Oxidative Treatments to a Human Colorectal Cancer Line. Journal of Food Biochemistry 2017, 41(2), Article number e12310. doi: 10.1111/ jfbc. 12310

15. Batra P., Sharma A.K. Anti-cancer potential of Flavonoids: recent trends and future perspectives. Biotech. 2013, 3(6), 439-459. doi: 10.1007/s13205-013-0117-5

16. Paul T., Ramasubbu S. The antioxidant, anticancer and anticoagulant activities of Acanthus ilicifolius L. roots and Lumnitzera racemosa Willd. leaves, from southeast coast of India. Journal of Applied Pharmaceutical Science 2017, 7(3), 81-87.

17. Nabavi S.F., Braidy N., Habtemariam S., Sureda A., Manayi A., Nabavi S.M. Neuroprotective Effects of Fisetin in Alzheimer's and Parkinson's Diseases: From Chemistry to Medicine. Current Topics in Medicinal Chemistry 2016, 16(17), 1910-1915. doi:10.2174/1568026 616666160204121725

18. Li J., Jiang Z., Li X., Hou Y., Liu F., Li N., Liu X., Yang L., Chen G. Natural therapeutic agents for neurodegenerative diseases from a traditional herbal medicine Pongamia pinnata (L.) Pierre. Bioorganic and Medicinal Chemistry Letters 2015, 25(1), 53-58. doi: 10.1016/j. bmcl.2014.11.015

19. Survay N.S., Upadhyaya C.P., Kumar B., Young K.E., Yoon D.-Y. Park, S.-W. New generation of flavonols and flavonol derivatives as therapeutic molecules. Journal of Applied Biological Chemistry 2011, 54(1), 1-18. doi: 10.3839/jksabc.2011.001

20. Youdim K.A., Dobbie M.S., Kuhnle G., Proteggente A.R., Abbott N.J., Rice-Evans C. Interaction between flavonoids and the blood-brain barrier: in vitro studies. Journal of Neurochemistry 2003, 85(1), 180-192. doi: 10.1046/j.1471-4159.2003.01652.x

21. Moosavi F., Hosseini R., Saso L., Firuzi O. Modulation of neurotrophic signaling pathways by polyphenols. Drug Design, Development and Therapy 2016, 10, 23-42. doi: 10.2147/DDDT. S96936

22. Rahal A., Kumar A., Singh V., Yadav B., Tiwari R., Chakraborty S., Dhama K. Oxidative Stress, Prooxidants, and Antioxidants: The Interplay. BioMed Research International 2014, 2014, 761264. doi: 10.1155/2014/761264

23. Di Meo S., Reed T.T., Venditti P., Victor V.M. Role of ROS and RNS Sources in Physiological and Pathological Conditions. Oxidative Medicine and Cellular Longevity 2016, 2016, 1245049. doi: 10.1155/2016/1245049

24. Kurutas E.B. The importance of antioxidants which play the role in cellular response against oxidative/nitrosative stress: current state. Nutrition Journal 2015, 15, 71. doi: 10.1186/s12937-016-0186-5 
25. Uttara B., Singh A.V., Zamboni P., Mahajan R.T. Oxidative stress and neurodegenerative diseases: a review of upstream and downstream antioxidant therapeutic options. Current Neuropharmacology 2009, 7(1), 65-74. doi: 10.2174/157015909787602823.

26. Rodrigues R., Petersen R.B., Perry G. Parallels between major depressive disorder and alzheimer's disease: role of oxidative stress and genetic vulnerability. Cellular and Molecular Neurobiology 2014, 34(7), 925-949. doi:10.1007/s10571-014-0074-5

27. Yan M.H., Wang X., Zhua X. Mitochondrial defects and oxidative stress in Alzheimer disease and Parkinson disease. Free Radical Biology \& Medicine 2013, 62, 90-101. doi: 10.1016/j.freeradbiomed.2012.11.014

28. Butterfield D.A., Swomley A.M., Sultana R. Amyloid $\beta$-Peptide (1-42)-Induced Oxidative Stress in Alzheimer Disease: Importance in Disease Pathogenesis and Progression. Antioxidants \& Redox Signaling 2013, 19(8), 823-835. doi: 10.1089/ars.2012.5027

29. Bendor J.T., Logan T.P., Edwards R.H. The Function of a-Synuclein. Neuron 2013, 79(6), 1044-1066. doi: 10.1016/j. neuron.2013.09.004

30. Dias V., Junn E., Mouradian M.M. The Role of Oxidative Stress in Parkinson's Disease. Journal of Parkinson's Disease 2013, 3(4), 461-491. doi: 10.3233/JPD-130230

31. Thilakarathna S.H., Rupasinghe H.P.V. Flavonoid Bioavailability and Attempts for Bioavailability Enhancement. Nutrients 2013, 5, 3367-3387. doi:10.3390/nu5093367

32. Kumar S., Pandey A.K. Chemistry and Biological Activities of Flavonoids: An Overview. ScientificWorldJournal 2013, 2013, 162750. doi:10.1155/2013/162750

33. Rangel-Ordonez L., Noldner M., Schubert-Zsilavecz M., Wurglics M. Plasma levels and distribution of Flavonoids in rat brain after single and repeated doses of standardized Ginkgo biloba Extract EGb 761. Planta Medica 2010, 76, 1683-1690. doi: 10.1055/s-0030-1249962

34. Rendeiro C., Rhodes J.S., Spencer J.P. The mechanisms of action of Flavonoids in the brain: Direct versus indirect effects. Neurochemistry International 2015, 89, 126-139. doi: 10.1016/j.neuint.2015.08.002

35. Lin C.C., Yang C.C., Chen Y.W., Hsiao L.D., Yang C.M. Arachidonic Acid Induces ARE/Nrf2-Dependent Heme Oxygenase-1 Transcription in Rat Brain Astrocytes. Molecular Neurobiology 2017, doi: 10.1007/ s12035-017-0590-7.

36. Bulboacă A., Bolboacă S.D., Suciu S. Protective effect of curcumin in fructose-induced metabolic syndrome and in streptozotocin-induced diabetes in rats. Iranian Journal of Basic Medical Sciences 2016, 19(6), 585-593.

37. Scapagnini G., Vasto S., Abraham N.G., Caruso C., Zella D., Fabio, G. Modulation of Nrf2/ARE pathway by food polyphenols: a nutritional neuroprotective strategy for cognitive and neurodegenerative disorders. Molecular Neurobiology 2011, 44(2), 192-201. doi: 10.1007/s12035-011-8181-5

38. Xi Y.D., Yu H.L., Ding J., Ma W.W., Yuan L.H., Feng J.F., Xiao Y.X., Xiao R. Flavonoids protect cerebrovascular endothelial cells through Nrf2 and PI3K from $\beta$-amyloid peptide-induced oxidative damage. Current Neurovascular Research 2012, 9(1), 32-41. doi: 10.2174/156720212799297092

39. Gopinath K., Sudhandiran G. Naringin modulates oxidative stress and inflammation in 3-nitropropionic acid-induced neurodegeneration through the activation of nuclear factor-erythroid 2-related factor-2 signalling pathway. Neuroscience 2012, 227, 134-143. doi: 10.1016/j. neuroscience.2012.07.060

40. Lou H., Jing X., Wei X., Shi H., Ren D., Zhang X. Naringenin protects against 6-OHDA-induced neurotoxicity via activation of the Nrf2/ARE signaling pathway. Neuropharmacology 2014, 79, 380-388. doi: 10.1016/j.neuropharm.2013.11.026.

41. Zhou Y., Xie N., Li L., Zou Y., Zhang X., Dong M. Puerarin alleviates cognitive impairment and oxidative stress in APP/PS1 transgenic mice. International Journal of Neuropsychopharmacology 2014, 17(4), 635-644. doi: 10.1017/S146114571300148X.

42. Kwon S.H., Ma S.X., Hwang J.Y., Lee S.Y., Jang C.G. Involvement of the $\mathrm{Nr} 2 / \mathrm{HO}-1$ signaling pathway in sulfuretin-induced protection against amyloid beta25-35 neurotoxicity. Neuroscience 2015, 304, 14-28. doi: 10.1016/j.neuroscience.2015.07.030.

43. Gaballah,H.H., Zakaria S.S., Elbatsh M.M., Tahoon N.M. Modulatory effects of resveratrol on endoplasmic reticulum stress-associated apoptosis and oxido-inflammatory markers in a rat model of rotenone-induced Parkinson's disease. Chemico-Biological Interactions 2016, 251, 10-6. doi: 10.1016/j.cbi.2016.03.023.

44. Shakeel S., Rehman M.U., Tabassum N., Amin U., Mir M.R. Effect of Naringenin (A naturally occurring flavanone) Against Pilocarpineinduced Status Epilepticus and Oxidative Stress in Mice. Pharmacognosy Magazine 2017, 13(Suppl 1), S154-S160. doi:10.4103/09731296.203977

45. Gao J., Inagaki Y., Liu Y. Research progress on flavonoids isolated from traditional Chinese medicine in treatment of Alzheimer's disease. Intractable \& Rare Diseases Research 2013, 2(1), 3-10. doi:10.5582/irdr.2013.v2.1.3

46. Ma M.W., Wang J., Zhang Q., Wang R., Dhandapani K.M., Vadlamudi R.K., Brann D.W. NADPH oxidase in brain injury and neurodegenerative disorders. Molecular Neurodegeneration 2017, 12, 7. doi:10.1186/s13024-017-0150-7

47. Hmidene B., Hanaki M., Murakami K., Irie K., Isoda H., Shigemori H. Inhibitory Activities of Antioxidant Flavonoids from Tamarix gallica on Amyloid Aggregation Related to Alzheimer's and Type 2 Diabetes Diseases. Biological and Pharmaceutical Bulletin 2017, 40(2), 238-241. doi:10.1248/bpb.b16-00801

48. Gutierrez-Zepeda A., Santell R., Wu Z., Brown M., Wu Y., Khan I., Link C.D., Zhao B., Luo Y. Soy isoflavone glycitein protects against beta amyloid-induced toxicity and oxidative stress in transgenic Caenorhabditis elegans. BMC Neuroscience 2005, 6, 54. doi:10.1186/1471-2202-6-54

49. Zhao L., Wang J.L., Liu R., Li X.X., Li J.F., Zhang L. Neuroprotective, anti-amyloidogenic and neurotrophic effects of apigenin in an Alzheimer's disease mouse model. Molecules 2013, 18, 9949-9965. doi:10.3390/molecules18089949

50. McCarty M.F. NADPH Oxidase Activity in Cerebral Arterioles Is a Key Mediator of Cerebral Small Vessel Disease-Implications for Prevention. Healthcare (Basel) 2015, 3(2), 233-251. doi:10.3390/ healthcare3020233

51. Huang T.C., Lu K.T., Wo Y.Y., Wu Y.J., Yang Y.L. Resveratrol protects rats from $A \beta$-induced neurotoxicity by the reduction of iNOS expression and lipid peroxidation. PLoS One. 2011;6(12):e29102. doi: 10.1371/journal.pone.0029102. Epub 2011 Dec 29

52. Duarte J., Francisco V., Perez-Vizcaino F. Modulation of nitric oxide by Flavonoids. Food \& Function 2014, 5(8), 1653-1668. doi:10.1039/ c4fo00144c. 